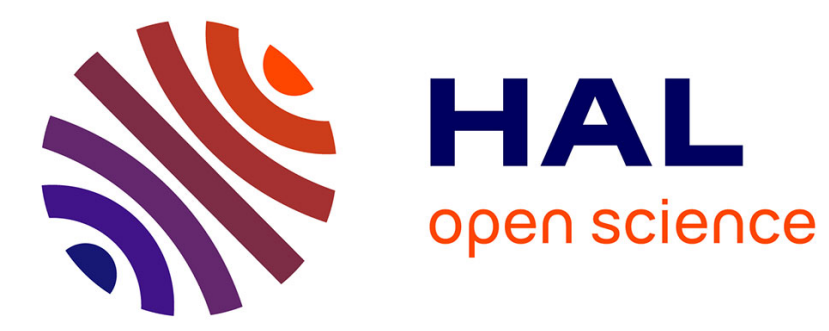

\title{
Automatic parallel parking and platooning to redistribute electric vehicles in a car-sharing application
}

Mohamed Marouf, Evangeline Pollard, Fawzi Nashashibi

\section{To cite this version:}

Mohamed Marouf, Evangeline Pollard, Fawzi Nashashibi. Automatic parallel parking and platooning to redistribute electric vehicles in a car-sharing application. IV'14 - IEEE Intelligent Vehicles Symposium, Jun 2014, Dearborn, Michigan, United States. hal-01018707

\section{HAL Id: hal-01018707 https://hal.inria.fr/hal-01018707}

Submitted on 4 Jul 2014

HAL is a multi-disciplinary open access archive for the deposit and dissemination of scientific research documents, whether they are published or not. The documents may come from teaching and research institutions in France or abroad, or from public or private research centers.
L'archive ouverte pluridisciplinaire HAL, est destinée au dépôt et à la diffusion de documents scientifiques de niveau recherche, publiés ou non, émanant des établissements d'enseignement et de recherche français ou étrangers, des laboratoires publics ou privés. 


\title{
Automatic parallel parking and platooning to redistribute electric vehicles in a car-sharing application
}

\author{
Mohamed MAROUF $^{1}$, Evangeline POLLARD ${ }^{1}$, Fawzi NASHASHIBI $^{1}$
}

\begin{abstract}
In car-sharing applications and during certain time slots, some parking parks become full whereas others are empty. To redress this imbalance, vehicle redistribution strategies must be elaborated. As automatic relocation cannot be in place, one alternative is to get a leader vehicle, driven by a human, which come to pick up and drop off vehicles over the stations. This paper deals with the vehicle redistribution problem among parking using this strategy and focuses on automatic parking and vehicle's platooning. We present an easy exit parking controller and path planning based only on geometric approach and vehicle's characteristics. Once the vehicle exits the parking, it joins a platoon of vehicles and follows it automatically to go to an empty parking space.
\end{abstract}

\section{INTRODUCTION}

Sustainable mobility leads to limit individual properties and to increase resource sharing. This is particularly true and realistic concerning urban transportation means, where bikes, motorbikes, cars and any new urban transportation systems [1] can be easily shared due to the high concentration of people. In Paris, for instance, the trend is to develop selfservice mobility services. With the bike sharing system velib, comprising 14000 bikes, 1200 stations and 225 000 subscribers, as well as the electric car-sharing system autolib, comprising 2000 vehicles, 1200 stations and 65 000 subscribers [2], Paris is definitively following this new mobility trend. Both velib and autolib systems are conceived as multiple station shared vehicle systems (MSSVS) [3] for short local trips (home to workplace, or home to the closest station for instance). In these systems, a group of vehicles is distributed among fixed stations. With MSSVS, round trips can occur but one-way trips as well, leading to a complicated fleet management. Indeed, the number of vehicles per station can quickly become imbalanced depending on the rush time and on the location (living areas vs. commercial areas). There are frequent disparities between the availability of rental vehicle and the number of rental slots. Relocation strategies are then useful to balance the number of vehicles and meet the demand. To solve this problem with the velib system, operators manually displace more than 3000 bikes daily, corresponding to $3 \%$ of the total fleet motion. For car-sharing system, relocation strategies are more difficult to implement. Various complicated strategies of relocation have been proposed in the past [4]: ride-sharing (two people travel in one vehicle to pick up another), equip vehicles with a hitch to tow another vehicle behind, using a scooter which will be towed back. However, all these strategies suffer from a lack

\footnotetext{
${ }^{1}$ The authors are at IMARA-INRIA Domaine de Voluceau - Rocquencourt B.P. 105 - 78153 Le Chesnay firstname. lastname at inria.fr
}

of time and energy efficiency. On the other hand, even if the tendency is to go towards automation opening new automatic relocation strategies, a fully automatic relocation, implying the movement of vehicles traveling without a driver on open roads, looks difficult for legal reasons. One alternative would have to get a leader vehicle with a driver and to regulate the number of vehicles over stations using platooning. In that way, the leader vehicle would act as an agent which would pick up and drop off vehicles over the stations. This solution looks more realistic in terms of safety and legacy.

In this article, we are not dealing with the problem of pickup and delivery which is largely tackled in the literature [5], [6]. We describe the implementation of a new system dedicated to an easy relocation using automatic parking and platooning for an electric car sharing application. Both perception, planing, control and communication issues are tackled in this article. A special attention will be given to the control aspects, parking maneuver and platooning staying challenging issues.

Many researches on parallel parking have been presented with different control approaches. These approaches can be divided into two categories: one based on stabilizing the vehicle to a target point, the other is based on path planning. Some controllers of the first group are based on Lyapunov function [7] where the function's parameters have to be hardly changed according to the free parking space. Other controllers are based on fuzzy logic [8], neurofuzzy control [9] and neural network [10]. These latter controllers need learning human skills which is limited and not easily extended to more general cases. The second group of controllers are based on path planning [11], [12]. These controllers plan a geometric collision-free path to park (res. retrieve) a vehicle in (resp. from) a parking slot. These controllers can demand heavy computations. For this reason, we present in this paper an easy way for path planning based on non-holonomic kinematic model of a vehicle.

Since the 70's, platooning has been studied to increase the throughput of roads. PATH in California [13] and PRAXITELE in France [14], [15] were the first pioneering projects. Later on, Auto21 [16] focused on the smooth merging and splitting of platoon considering only highways for platooning-enabled cars. In SARTRE project [17], platoons are considered fully autonomous except for the leading vehicle, which will be driven manually, while all other vehicles are free to join and leave the platoon. A model of platooning vehicles with a constant inter-vehicle spacing has been presented in [18].

The paper is organized as follows. In Sec. II, a global 
description of the system elements is provided. In Sec. III, the perception issues are described. In Sec. IV, the innovate strategies for platooning and automatic parking are explained. Finally, in Sec. V, experimental results are presented, before we conclude in Sec. VI.

\section{SYSTEM DESCRIPTION}

The general architecture of the relocation system is shown in Fig. 1. A supervisor centralizes positions of the fleet vehicles. From this information, it will calculate the operatorbased relocation strategy, i.e. finding the best fleet distribution over stations to maximize the system performances. If necessary, missions orders are sent to the operators through communication knowing the maximum number of vehicles into a platoon. Missions consist in picking up one or several vehicles from an overloaded station and drop them off at one or several empty stations. The operator can follow the accomplishment of its current mission through the Human Machine Interface (HMI). Its accomplishment is also sent to the supervisor.

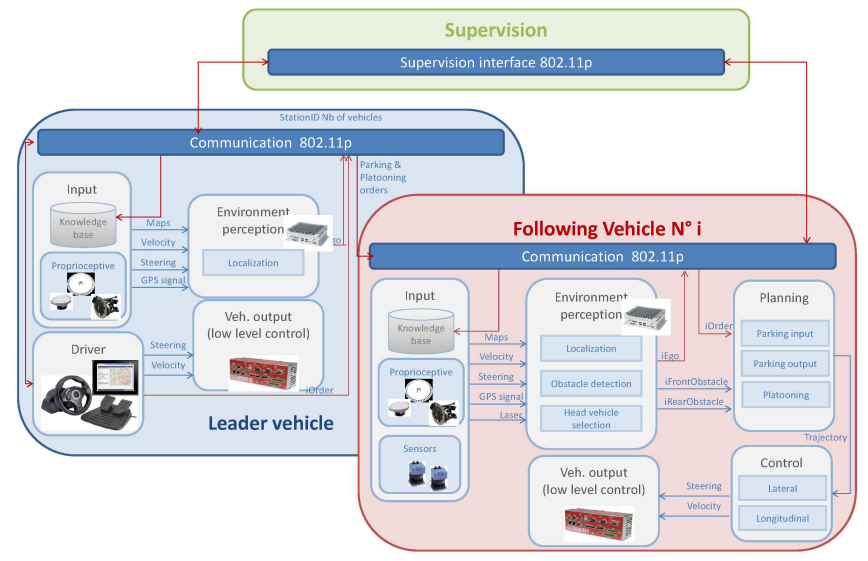

Fig. 1. System architecture

The leader vehicle can be any automotive vehicle, equipped with communication devices and localization means. The driver sends orders through a HMI delivered on a tablet. To help the driver, global planning (itinerary calculation) is made using online maps. In our application, the shared electric vehicles are equipped with many sensors (Lidar, GPS, etc.) in order to observe their environment and localize themselves, with computers to process these data, with actuators to command the vehicle and with $\mathrm{Hu}$ man Machine Interface to interact with the driver. Sensor configuration is shown in Fig. 2. As data coming from sensors are noisy, inaccurate and can also be unreliable or unsynchronized, the use of data fusion techniques is required in order to provide the most accurate situation assessment as possible. For this application, situation assessment consists in merging information about the vehicle state by itself (position, velocity, acceleration, battery level, etc.) to accurately localize the vehicle; in detecting potential obstacles like other vehicles, bicycles or pedestrians. Local planning is made to calculate the vehicle path according to the scenario (parking input/output, platooning) and the corresponding commands for the lateral and longitudinal control are sent to the lowlevel controller.

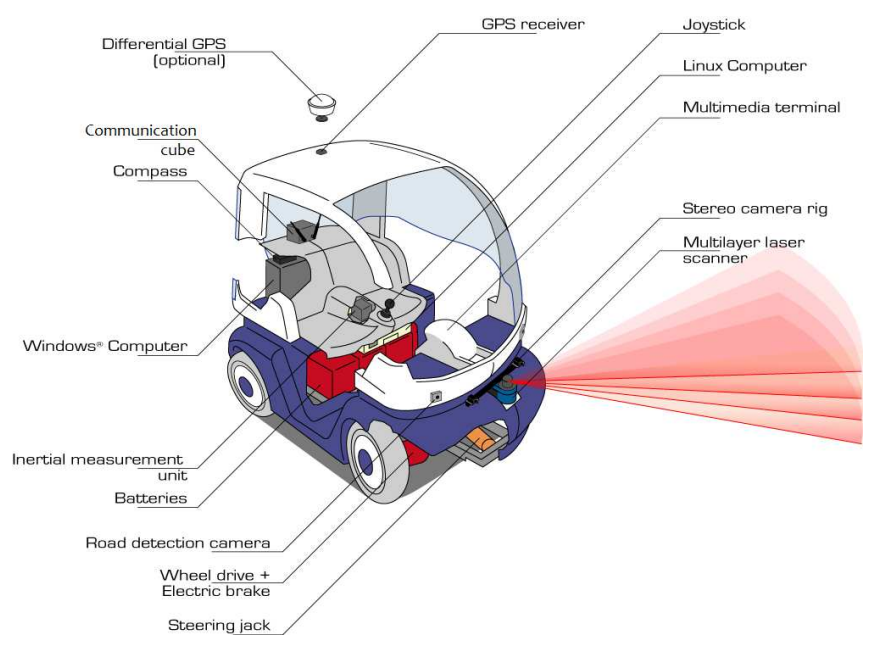

Fig. 2. Sensors and actuators of the electric vehicle Cycab

When the head vehicle of a platoon receives a mission order to pick up a shared vehicle localized with GPS position, the platoon moves to that position and stop in such a way that the tail vehicle of the platoon will be in front of the parked vehicle in order to let it exit the parking. The platoon's heads vehicle communicate with the parked vehicle to start the exit parking maneuver. The parked vehicle starts to exit the parking. Once this maneuver finished, it detects the last vehicle of the platoon and join the platoon, then it acknowledges the head vehicle that the exit parking maneuver is finished and it becomes the tail vehicle. The head vehicle updates the new platoon configuration and send it to the supervisor. Once the platoon arrives to the empty parking, it stops and send order to the platoon vehicles to park one by one. When all shared vehicles are parked or the parking becomes full, the head vehicle updates its configuration and send it to the supervisor, then continues following the supervisor orders.

\section{PERCEPTION ISSUES}

Relatively to this automatic parking and platooning application, the perception task is reduced to the obstacle detection and object tracking as it is described in the section below.

\section{A. Multi object detection and tracking in 5 steps}

In the Data processing step (1), distances coming from the front and rear laser sensors are converted into $(x, y, z)$ points in the local Cartesian coordinate system. They are then sorted depending on their angle to the coordinate system center. In the Segmentation step (2), a Cluster-based Recursive Line fitting algorithm is used with parameter $d_{1}^{\prime}$ and $d_{2}^{\prime}$ for the maximum distances to the closest segment and between two successive segments respectively (see [19]). In the Clustering step (3), segments are associated to create objects. Considering our parking application, close obstacles are considered 
and objects with less than 5 laser impacts are filtered. In the Classification step (4), size and shape consideration are used to obtain a raw classification of the object. In the Tracking step (5), information about the ego-vehicle dynamics are considered (velocity and steering angle) to improve the tracking of the object in the local Cartesian coordinate system. Object tracking is done in relative coordinates regarding the egovehicle using a Constant Velocity Kalman filter and Nearest Neighbor approach for data association.

\section{B. Head/Rear vehicle selection}

During the exit parking maneuver, closest front and rear cars are selected to calculate front and back distances. In case a pedestrian or any smaller obstacle is detected around the ego-vehicle, an emergency stop is applied. Then, for the platooning input, the front vehicle is detected as a vehicle, following a car shape, which is the closest obstacle in a corridor surrounding the vehicle path.

\section{CONTROL STRATEGIES}

We first present the parallel exit parking controller which allows retrieving a vehicle from a parking slot to be at the tail of the platoon. Then, we present the platooning longitudinal and lateral controllers. The parallel parking controller is not specifically described because it is the exact reverse maneuver in comparison to the parallel exit parking controller.

\section{A. Exit parking controller}

The aim of this controller is to retrieve a vehicle from its parking slot to a final position which is parallel to its initial position. The closed loop of this controller is given by Fig. 3. Front and back lasers are used to detect the front and back vehicles, and other obstacles to be avoided, and as we need a relative position and direction, we used an odometer model based on rear wheels incremental encoder sensors.

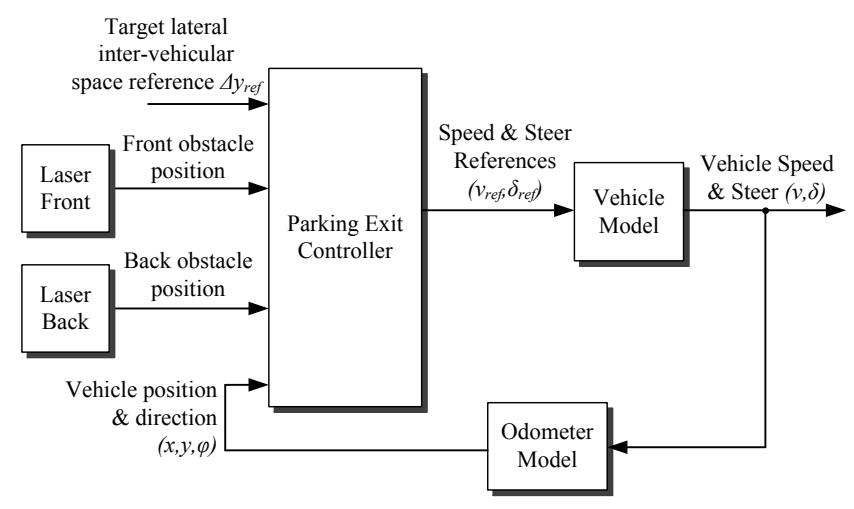

Fig. 3. Functional Structure of Parking Exit Controller

1) Vehicle geometry and path planning: As shown in Fig. 4, a vehicle is characterized by its width $w$ and its length $L$ which is composed of a wheelbase $l$ and respectively a front and back overhang $e_{f}$ and $e_{b}$. When a vehicle is moving with a constant steering angle $\delta$, it generates a circular trajectory of a radius $R$. Also, the vehicle is moving between an inner circle of radius $R_{i}$ and an outer circle of radius $R_{o}$. All these circles have the same center $C\left(x_{c}, y_{c}\right)$. When the vehicle is moving with its maximum steering angle $\delta_{\max }$, we obtain the minimum values of the previous defined radii. $R_{\text {min }}, R_{i \min }$ and $R_{o \text { min }}$ are respectively given by:

$$
\begin{gathered}
R_{\text {min }}=\frac{l}{\tan \left(\delta_{\max }\right)} \\
R_{\text {imin }}=R_{\text {min }}-\frac{w}{2}=\frac{l}{\tan \left(\delta_{\max }\right)}-\frac{w}{2}
\end{gathered}
$$

By using Pythagorean theorem we obtain:

$$
R_{\text {omin }}=\sqrt{\left(R_{\text {min }}+\frac{w}{2}\right)^{2}+\left(l+e_{f}\right)^{2}}
$$

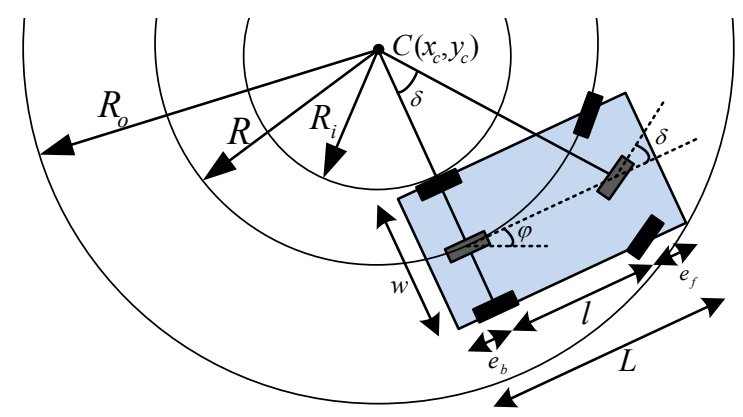

Fig. 4. Geometry of a vehicle turning with a constant steering angle $\delta$

The exit parking can be done in one trial ( $c f$. Fig. 5 and 6) or many trials ( $c f$. Fig. 7).

For one trial maneuver, the vehicle needs enough space between the ego and the front parked vehicle. In this case, the vehicle's trajectory is combined by two tangent circular arcs connected by the turning point. The minimum space allowing one trial maneuver is determined by the static vehicle's back left edge point $E\left(x_{e}, y_{e}\right)$ ( $c f$. Fig. 6). This latter must be outside the circle of radius $R_{o \text { min }}$ and center $C_{1}$, and the minimum space $S_{\min }$ corresponds to the distance between the ego vehicle and the point of the outer circle having $y$ coordinate equal to $y_{e}$ as shown in Fig. 6. In this case, the $x$ coordinate of this point is given by $\sqrt{R_{o \text { min }}^{2}-\left(y_{1}-y_{e}\right)^{2}}$ which gives

$$
S_{\text {min }}=\sqrt{R_{o \text { min }}^{2}-\left(R_{m i n}-y_{e}\right)^{2}}
$$

If the free space between vehicles is less than $S_{m i n}$, the vehicle has to do many maneuvers as shown in Fig. 7. The first maneuver is to move backward until it reaches a secure distance between vehicles, then we propose a bangbang controller based on the minimum spacing computation relatively to the vehicle mobile referential. However, $S_{\min }$ is always changing because $y_{e}$ will change when the vehicle moves.

If $x_{e}<S_{\min }$, then the vehicle moves forward with $\delta=+\delta_{\max }$ until reaching a secure distance between the ego vehicle and the front vehicle. Then it moves backward 


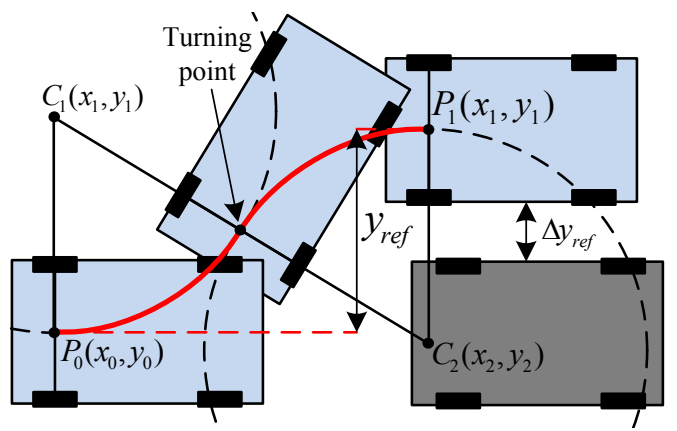

Fig. 5. Parallel Parking Exit for one maneuver case

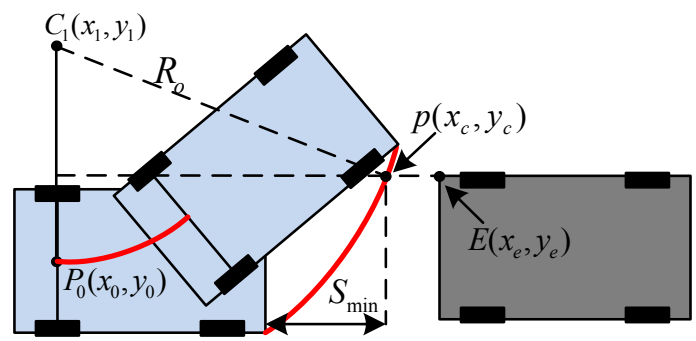

Fig. 6. One trial maneuver

with $\delta=-\delta_{\max }$ until reaching a secure distance between the ego vehicle and the back vehicle. However, during this maneuver, if $x_{e}<S_{\min }$ then the vehicle moves forward with $\delta=+\delta_{\max }$ until reaching the turning point. After, the vehicle continue moving forward but with $\delta=-\delta_{\max }$ until it becomes parallel to its initial position.

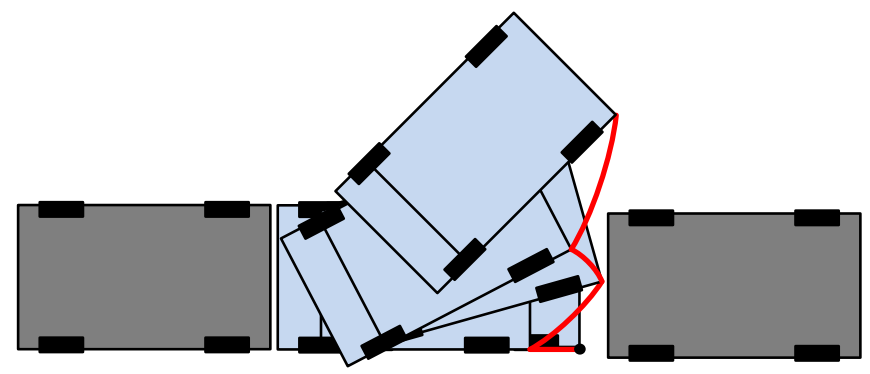

Fig. 7. Many trials maneuvers

The turning point is computed according to the vehicle's head angle $\phi$. This later must be tangent to a circle of a radius $R_{\text {min }}$ and a center $C_{2}\left(x_{2}, y_{2}\right)$. The coordinate $y_{2}$ is defined by:

$$
y_{2}=y_{r e f}-R_{\min } .
$$

For each vehicle's position $P_{i}$, we define a distance $r_{i}$ as shown in Fig. 8. This distance is equal to $R_{\min }$ when the vehicle is tangent to the circle in the turning point, for instance, in Fig. 8, $P_{3}$ is the turning point. In the general case, the distance $r$ is given by

$$
r=\frac{y+\left(R_{\min }-y_{r e f}\right)}{\cos (\phi)} .
$$

The turning point is reached if $r_{i} \geq R_{\min }$ which means

$$
y \geq y_{\text {ref }}-R_{\min }(1+\cdot \cos (\phi)) .
$$

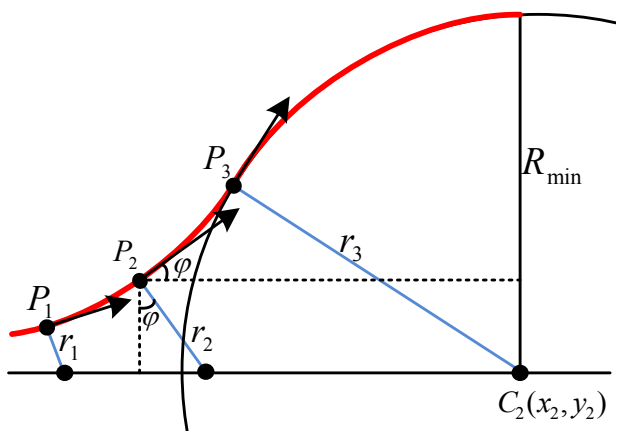

Fig. 8. Turning point computation

\section{B. Platooning controller}

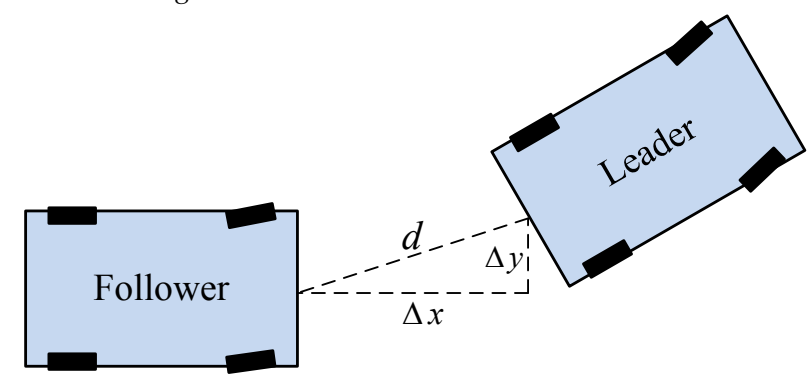

Fig. 9. Platoon of two vehicles

Once the vehicle finishes the exit parking maneuver, it detects the tail vehicle of the platoon and initializes the laser tracking algorithm to track this vehicle with a constant inter-vehicle spacing of 2 meters $\left(d_{r e f}\right)$. The tracking algorithm calculates the relative front vehicles position $\left(X_{r e l}, Y_{r e l}, \theta_{r e l}\right)$ relatively to the ego vehicle.

We use a classical proportional integral controller to control the velocity $\nu(t)$ of the follower vehicle. For a measured inter- distance $d(t)$, the velocity of the follower vehicle is given by:

$$
\nu(t)=K_{p} \cdot e(t)+K_{i} \cdot \int_{0}^{t} e(\tau) \mathrm{d} \tau
$$

where $K_{p}$ and $K_{i}$ are respectively the proportional and the integral gains, and the error $e(t)$ is given by:

$$
e(t)=d_{r e f}-d(t)
$$

The proposed lateral controller is based on a constant curvature approach, which allows the follower vehicle movement from its initial position $\left(X_{F}, Y_{F}\right)$ to the leader vehicle's position $\left(X_{L}, Y_{L}\right)$ with a constant steer angle $\delta$. The steer angle $\delta$ is given by :

$$
\delta=\operatorname{atan}\left(\frac{2 \cdot l \cdot \sin (\phi)}{\sqrt{\Delta x^{2}+\Delta y^{2}}}\right)
$$




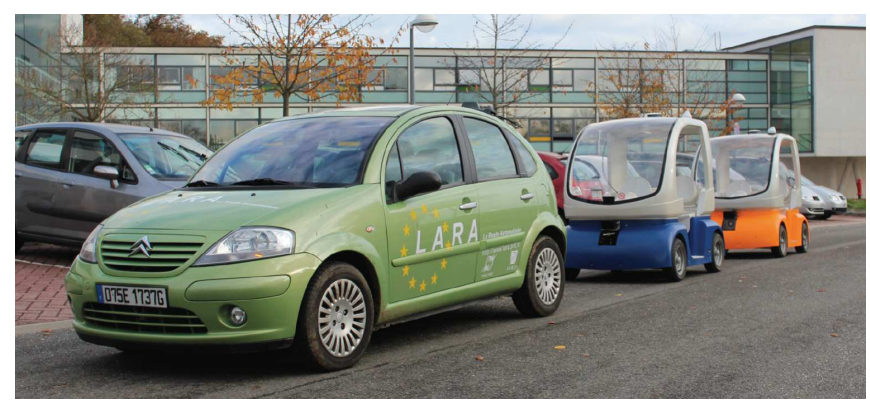

Fig. 10. Three vehicles platoon demonstration

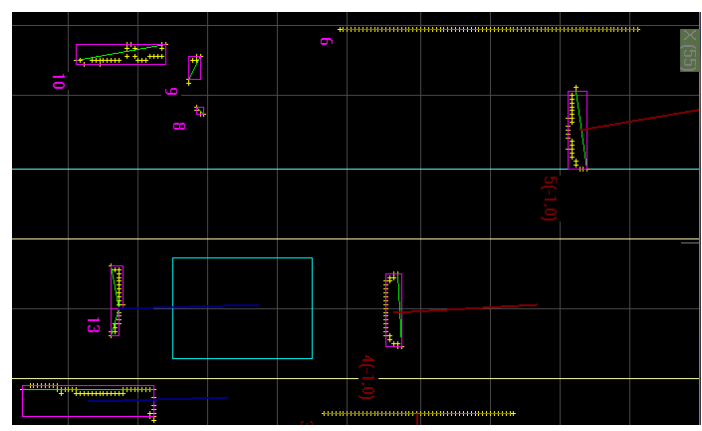

Fig. 11. Laser objects detection

where

$$
\left\{\begin{array}{l}
\phi=\operatorname{atan}\left(\frac{\Delta y}{\Delta x}\right)-\theta \\
\Delta x=X_{L}-X_{F} \\
\Delta y=Y_{L}-Y_{F}
\end{array}\right.
$$

For more informations see [20].

\section{EXPERIMENTAL RESULTS}

Our approach has been implemented and tested on our experimental electric vehicles called Cycab. They are equipped by an embedded fanless PC having an Intel Core i7 Pentium and running under Windows 764 bits operating system. We implemented high-level exit parking and platooning controller using RTMaps software [21]. This high-level controller communicates with the low-level controllers using a CAN bus. The low-level controllers are PID speed and steering controllers implemented on MPC555 microcontrollers to control 4 DC motors for propulsion and an electric jack for steering using Curtis power drivers. We also used two Hokuyo UTM-30LX lasers having $30 \mathrm{~m}$ and $270^{\circ}$ scanning range. The head vehicle can be any manually driven vehicle, in our case it is a Citroën C3 vehicle. Both head and follower vehicles are equipped with communication cubes enabling low latency communication, and considering vehicular communication standard IEEE $802.11 p$ [22].

Fig. 11 shows the laser impact points and the detected objects of one electric vehicle parked between two other vehicles, and a tail platoon's vehicle waiting the parked vehicle to exit the parking slot and join the platoon. The parked vehicle is represented by a blue rectangle. Front and back parked vehicles are identified objects with IDs respectively equal to 4 and 13 , where the last vehicle of the platoon has an ID equal to 5 . There is almost $1 \mathrm{~m}$ space between the parked vehilces, and we also considered a secure distance of about $20 \mathrm{~cm}$. Other detected objects are considered as obstacles.

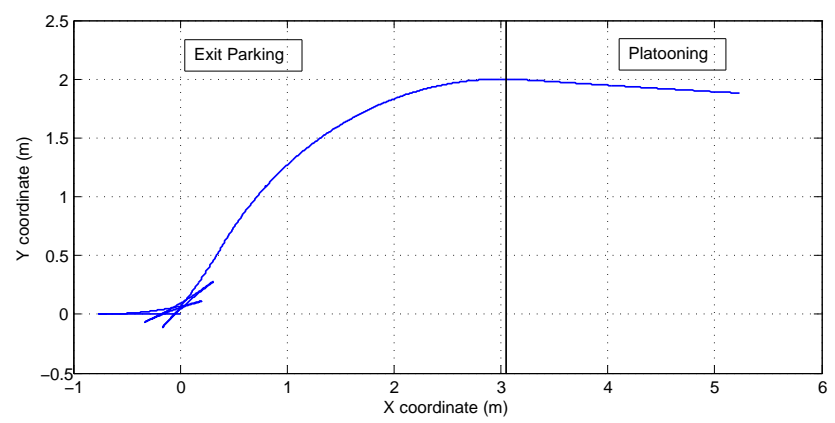

Fig. 12. Trajectory of exit parking and platooning

Fig. 12 shows the trajectory of the exit parking and the platooning. The left part of the figure shows the exit parking trajectory. As there was not enough space for one trial maneuver, the exit parking has been done in six maneuvers. At the end of the last maneuver, the vehicle become parallel to its initial position, it stops then detects the front vehicle to be tracked. Once done, it switches to the platooning mode and acknowledges the head vehicle to keep moving. The right part of Fig.12 shows the trajectory of the vehicle following the platoon.

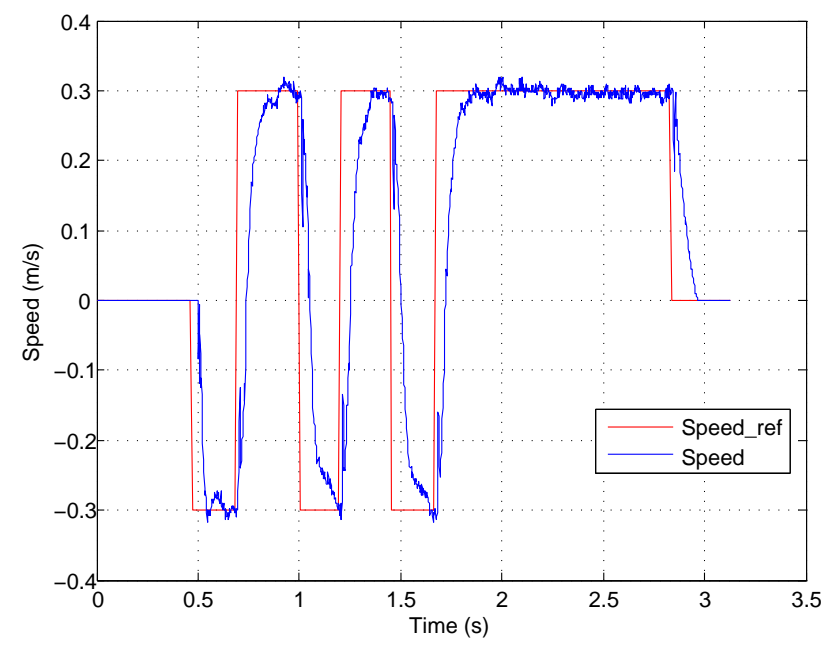

Fig. 13. Vehicle and reference velocities

Fig. 13 shows the reference velocity $\nu_{r e f}$ and the measured velocity $\nu$. The reference velocity is given by the bangbang controller explained in section IV-A. $\nu_{\text {ref }}$ switches six times between $+0.3 \mathrm{~m} / \mathrm{s}$ and $-0.3 \mathrm{~m} / \mathrm{s}$ which corresponds to the trajectory given by Fig. 12. The velocity $\nu$ follows the velocity reference $\nu_{r e f}$ and have a response time of about $0.1 \mathrm{~s}$.

Fig. 14 shows the reference steering angle $\delta_{\text {ref }}$ and the measured steering angle $\delta$. The reference steering angle switches six times between $+0.4 \mathrm{rad}$ and $-0.4 \mathrm{rad}$ which corresponds to the trajectory of the exit parking. The measured steering angle $\delta$ is following the reference steering 


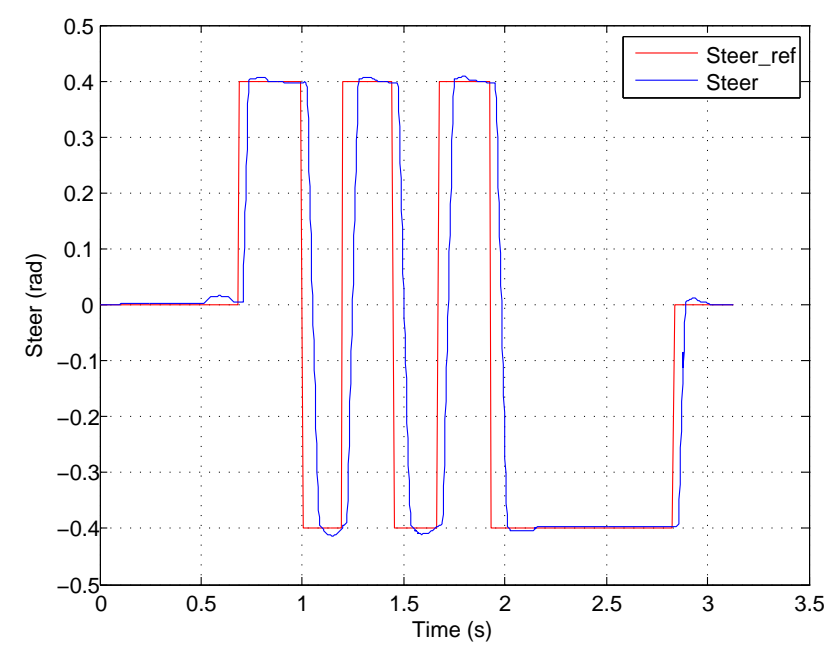

Fig. 14. Vehicle and reference steers

angle. We notice that the curve of $\delta$ is smooth because the driver is an electric jack. Also, there is a delay of about $0.03 \mathrm{~s}$ which is due to a mechanical gap between the electric jack and the steering rod. The response time including the delay is about $0.1 \mathrm{~s}$.

\section{Remark}

The response times and the delays presented below are also due to the low-level controller's period which is $10 \mathrm{~ms}$ and the CAN communication delay between the high-level and the low-level controllers.

\section{CONCLUSION}

In this paper, we described the implementation of a relocation strategy to regulate the number of cars in several car parks for a car-sharing application. The idea is to get a leader vehicle with a driver, which comes to pick up and drop off cars (without drivers) over stations using automatic parking and platooning. The path planing for the automatic vehicles is based on a non-holonomic kinematic model of the vehicle, which is easily implemented and really efficient. This has been demonstrated over several experimental results.

Perspectives, now, consists in implementing such a relocation strategy including the pickup and delivery problem and the supervisor communication to get a complete system for vehicle redistribution. Then, concerning platooning in urban areas, even if the legislation indicates that a platoon of vehicles in France follows the legal rules dedicated to little train for tourists, there is no doubt that specific strategies should be employed for urban platoon driving.

\section{REFERENCES}

[1] E. M. Cepolina and A. Farina, "A new shared vehicle system for urban areas," Transportation Research Part C: Emerging Technologies, vol. 21, no. 1, pp. 230 - 243, 2012. [Online]. Available: http://www.sciencedirect.com/science/article/pii/S0968090X11001495

[2] M. Huré and O. Waine. From Vélib to Autolib: private corporations involvement in urban mobility policy. [Online]. Available: http://www.metropolitiques.eu/From-Velib-to-Autolib-private.html

[3] E. Cepolina and A. Farina, "Urban car sharing: an overview of relocation strategies," WIT Transactions on the Built Environment, vol. 128, pp. 419-431, 2012.
[4] M. Barth and M. Todd, "Intelligent transportation system architecture for a multi-station shared vehicle system," in Intelligent Transportation Systems, 2000. Proceedings. 2000 IEEE, 2000, pp. 240-245.

[5] M. Dror, D. Fortin, and C. Roucairol, "Redistribution of Self-service Electric Cars: A Case of Pickup and Delivery," INRIA, Rapport de recherche RR-3543, 1998, projet PRAXITELE. [Online]. Available: http://hal.inria.fr/inria-00073142

[6] A. G. Kek, R. L. Cheu, Q. Meng, and C. H. Fung, "A decision support system for vehicle relocation operations in carsharing systems," Transportation Research Part E: Logistics and Transportation Review, vol. 45, no. 1, pp. 149 - 158, 2009. [Online]. Available: http://www.sciencedirect.com/science/article/pii/S1366554508000720

[7] S. Lee, M. Kim, Y. Youm, and W. Chung, "Control of a car-like mobile robot for parking problem," in Robotics and Automation, 1999. Proceedings. 1999 IEEE International Conference on, vol. 1, 1999, pp. 1-6 vol.1.

[8] R. Cabrera-Cosetl, M. Mora-Alvarez, and R. Alejos-Palomares, "Selfparking system based in a fuzzy logic approach," in Electrical, Communications, and Computers, 2009. CONIELECOMP 2009. International Conference on, 2009, pp. 119-124.

[9] Z.-L. Wang, C.-H. Yang, and T.-Y. Guo, "The design of an autonomous parallel parking neuro-fuzzy controller for a car-like mobile robot," in SICE Annual Conference 2010, Proceedings of, 2010, pp. 2593-2599.

[10] M. Heinen, F. Osorio, F. Heinen, and C. Kelber, "Seva3d: Using arti cial neural networks to autonomous vehicle parking control," in Neural Networks, 2006. IJCNN '06. International Joint Conference on, 2006, pp. 4704-4711.

[11] H. Vorobieva, N. Minoiu-Enache, S. Glaser, and S. Mammar, "Geometric continuous-curvature path planning for automatic parallel parking," in Networking, Sensing and Control (ICNSC), 2013 10th IEEE International Conference on, 2013, pp. 418-423.

[12] B. Muller, J. Deutscher, and S. Grodde, "Continuous curvature trajectory design and feedforward control for parking a car," Control Systems Technology, IEEE Transactions on, vol. 15, no. 3, pp. 541-553, 2007.

[13] S. Shladover, "Path at 20 - history and major milestones," in Intelligent Transportation Systems Conference, 2006. ITSC '06. IEEE, 2006, pp. 122-129.

[14] P. Daviet and M. Parent, "Longitudinal and lateral servoing of vehicles in a platoon," in Intelligent Vehicles Symposium, 1996., Proceedings of the 1996 IEEE, 1996, pp. 41-46.

[15] C. Laugier, "Towards autonomous vehicles for future intelligent transportation systems," in Proc. 6th Conf. of Italian Association in Artificial Intelligence. Citeseer, 1998, pp. 251-258.

[16] S. Hall, B. Chaib-draa, and J. Laumonier, "Car platoons simulated as a multiagent system," in In: Proc. 4th Workshop on Agent-Based Simulation, 2003, pp. 57-63.

[17] E. C. Tom Robinson, Eric Chan, "Operating platoons on public motorways: An introduction to the sartre platooning programme," in 17th World Congress on Intelligent Transport Systems (ITS) 2010, October 2010.

[18] P. Fernandes and U. Nunes, "Platooning with dsrc-based ivc-enabled autonomous vehicles: Adding infrared communications for ivc reliability improvement," in Intelligent Vehicles Symposium (IV), 2012 IEEE, 2012, pp. 517-522.

[19] P. Resende, E. Pollard, H. Li, and F. Nashashibi, "Low Speed Automation: technical feasibility of the driving sharing in urban areas," in IEEE ITSC 2013 - 16th International IEEE Conference on Intelligent Transportation Systems, La Hague, Netherlands, Jul. 2013.

[20] M. Abualhoul, M. Marouf, O. Shagdar, and F. Nashashibi, "Platooning Control Using Visible Light Communications: A Feasibility Study," in IEEE ITSC 2013, Hague, Netherlands, Mar. 2013. [Online]. Available: http://hal.inria.fr/hal-00835804

[21] "Intempora," http://www.intempora.com.

[22] S. Eichler, "Performance evaluation of the ieee 802.11p wave communication standard," in Vehicular Technology Conference, 2007. VTC2007 Fall. 2007 IEEE 66th, Sept 2007, pp. 2199-2203. 